\title{
ANALYSIS OF GEODESICS ON DIFFERENT SURFACES
}

\author{
MIROSLAV MAKSIMOVIĆ ${ }^{1, \star}$, TANJA JOVANOVIĆ ${ }^{1}$, EUGEN LJAJKO $^{1}$, MILICA IVANOVIĆ $^{1}$
}

${ }^{1}$ Faculty of Natural Sciences and Mathematics, University of Priština, Kosovska Mitrovica, Serbia

\begin{abstract}
It is widely known that some surfaces contain special curves as a geodesics, while a lots of geodesics on surface have complicated shapes. Goal of this research is to find on what surfaces are $u$ - and $v$ - parameter curves geodesics. Developable surfaces that contain a given plane curve as a geodesic are studied in the article, whereas the plane curve is also an initial $u$-parameter curve on that surface. Parametric equations of the minimal surfaces that contain an epicycloid as a geodesic are also given. Visualization of geodesics was done in Mathematica.
\end{abstract}

Keywords: Geodesics, $u$ - and $v$ - parameter curve, Developable surface, Minimal surface.

\section{INTRODUCTION}

Geodesics are those curves on the surface that are not "geodesically curved". Considering their role on surfaces, they can be compared to straight lines in plane and they are called "the most straight lines" on surface. A geodesic can be obtained as the solution of the nonlinear system of second-order differential equations with the given points and its tangent direction for the initial conditions. This system can be solved in special cases for plane surface, revolution surface and ruled surface. But, in the general case, for other surfaces it is difficult to solve this system. In Khuangsatung (2012) geodesics are presented and classified by Clairauts relation on some surfaces of revolution. For example, at Hyperboloid just the initial parallel is a geodesic and any other meridian is geodesic. That means they are actually initial $u$ - and $v$-parameter curve that are geodesics. In articles Abdel-All (2013) and Lewis (2002) it is shown that this system can be solved numerically for the given initial conditions, and for this purpose a Mathematica package code was written, that solves the system and shows obtained geodesics on the surfaces.

\section{NOTATION AND PRELIMINARIES}

Let $r: D \rightarrow \mathbb{R}^{3},(u, v) \mapsto(x(u, v), y(u, v), z(u, v))$ denote a mapping of an open set $D \subset \mathbb{R}^{2}$.

Definition 1. (Abbena (2006)) Let us give a surface $\mathcal{S}: r=r(u, v)$. For the point $\left(u_{0}, v_{0}\right) \in D$, curves

$$
u \rightarrow r\left(u, v_{0}\right) \text { and } v \rightarrow r\left(u_{0}, v\right)
$$

are called $u$-parameter curve and $v$-parameter curve on the surface $\mathcal{S}$.

For surface $\mathcal{S}$ denote with $E=r_{u} \cdot r_{u}, F=r_{u} \cdot r_{v}, G=r_{v} \cdot r_{v}$ coefficients of the first fundamental form

$$
I=E d u^{2}+2 F d u d v+G d v^{2}
$$

Gaussian curvature $K$ and mean curvature $H$ of the surface $\mathcal{S}$ are given with formulas

$$
K=\frac{L N-M^{2}}{E G-F^{2}}, \quad H=\frac{E N-2 F M+G L}{2\left(E G-F^{2}\right)} .
$$

A surface with $H=0$ is called minimal surface. A surface with $K=0$ is called developable surface.

Definition 2. (Abbena (2006)) The Christoffel symbols $\Gamma_{j k}^{i}$, $i, j, k=1,2$, of a regular surface $\mathcal{S}$ are given by

$$
\begin{array}{lrl}
\Gamma_{11}^{1}=\frac{G E_{u}-2 F F_{u}+F E_{v}}{2\left(E G-F^{2}\right)}, & \Gamma_{11}^{2}=\frac{2 E F_{u}-E E_{v}-F E_{u}}{2\left(E G-F^{2}\right)}, \\
\Gamma_{12}^{1}=\frac{G E_{v}-F G_{u}}{2\left(E G-F^{2}\right)}, & \Gamma_{12}^{2}=\frac{E G_{u}-F E_{v}}{2\left(E G-F^{2}\right)}, \\
\Gamma_{22}^{1}=\frac{2 G F_{v}-G G_{u}-F G_{v}}{2\left(E G-F^{2}\right)}, & \Gamma_{22}^{2}=\frac{E G_{v}-2 F F_{v}+F G_{u}}{2\left(E G-F^{2}\right)},
\end{array}
$$

where is $\Gamma_{12}^{1}=\Gamma_{21}^{1}$ and $\Gamma_{12}^{2}=\Gamma_{21}^{2}$.

For geodesic its said that it is the path of particles without acceleration and it is not affected by any outside factors, but it accelerates perpendicularly to the surface. According to that, we give the following definition.

Definition 3. (Pressley (2012)) Let $r(t)=r(u(t), v(t))$ be a smooth curve on a surface $\mathcal{S}$. The curve $r(t)$ is called a geodesic if $r^{\prime \prime}(t)$ is zero or perpendicular to the tangent plane of the surface at the point $r(t)$, for all values of the parameter $t$.

Definition 3 implies that $r^{\prime \prime}(t)$ and the normal to the surface are parallel.

Theorem 4. (Pressley (2012)) A curve $r(t)=r(u(t), v(t))$ on a surface $\mathcal{S}$ is a geodesic if and only if, for any part of $r(t)$ contained in a surface patch of $\mathcal{S}$, the following two equations are satisfied

$$
\left\{\begin{array}{l}
u^{\prime \prime}+\Gamma_{11}^{1} u^{\prime 2}+2 \Gamma_{12}^{1} u^{\prime} v^{\prime}+\Gamma_{22}^{1} v^{\prime 2}=0 \\
v^{\prime \prime}+\Gamma_{11}^{2} u^{\prime 2}+2 \Gamma_{12}^{2} u^{\prime} v^{\prime}+\Gamma_{22}^{2} v^{\prime 2}=0 .
\end{array}\right.
$$


where $\Gamma_{j k}^{i}$ a Christoffel symbols.

Theorem 5. (Do Carmo (1976)) Through any surface point in each direction passes one and only one geodesic.

This theorem tells us that each geodesic is determined by initial point and the speed in that point. Any geodesics has a constant speed, and, taking that into consideration, any straight line, or its part is a geodesics on a surface. This result gives us a motivation for some researches in this work.

Theorem 6. (Do Carmo (1976)) On the surface of revolution

$$
r(u, v)=(f(u) \cos v, f(u) \sin v, g(u)),
$$

every meridian $\left(v=v_{0}\right)$ is a geodesic and parallel $u=u_{0}$ is a geodesic if and only if $u_{0}$ is a stationary point of a function $f(u)$.

More about geodesics can be found in Do Carmo (1976), Abbena (2006), Pressley (2012) and Toponogov (2006).

\section{$u$ - AND $v$ - PARAMETER CURVES AS GEODESICS}

In this research we came to a conclusion that for some surfaces initial $u$ - and $v$-parameter curve satisfies conditions of geodesics. We are going to use red color to mark a $u$ - parameter curve, blue for a $v$-parameter curve and green for other geodesics.

\section{Eight surface}

Eight surface is a surface of revolution with parametrization

$$
r(u, v)=(\sin (2 u) \cos v, \sin (2 u) \sin v, \sin u) .
$$

Based on theorem 6. it is clear that its meridians will be geodesic. Solutions of the equation $f^{\prime}(u)=0$ are $u_{1,2}= \pm \pi / 4$, which means that the Eight surface has two parallels , $u_{1}=\pi / 4$ and $u_{2}=-\pi / 4$, that satisfy conditions for geodesics (fig. 1. a)). Other geodesics on the Eight surface "stay" only on one of its parts (fig. 1. b)), so the meridians are only geodesic of this surface that contains the coordinate origin.

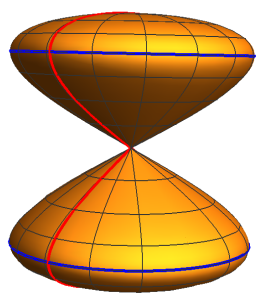

a)

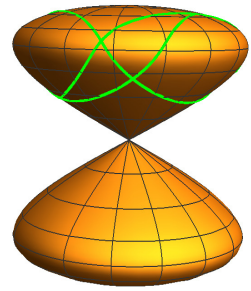

b)
Figure 1. Geodesics on Eight surface.

\section{Two banana surface}

Two banana surface is parametrized by

$$
r(u, v)=((2+\cos u) \cos v, \sin u \cos v, \sin v) .
$$

On the Two banana surface initial $u$ - and $v$-parameter curves are geodesics (fig. 2 a)). Other geodesics stay on one banana and have complicated shapes (Lewis (2002)) (fig. 2 b)).

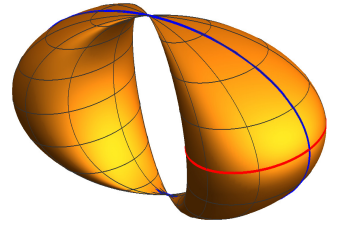

a)

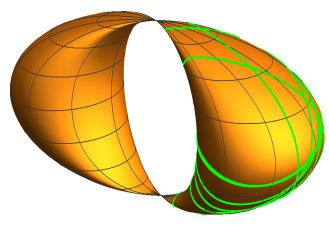

b)
Figure 2. Geodesics on Two banana surface.

\section{Monkey saddle}

Geodesics on monkey saddle, which has parametrization $r(u, v)=$ $\left(u, v, u^{3}-3 u v^{2}\right)$, are shown in fig. 3 .

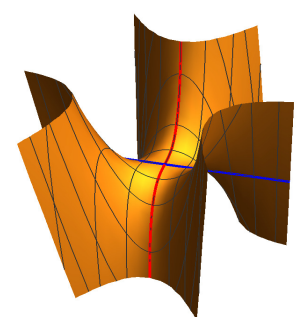

a)

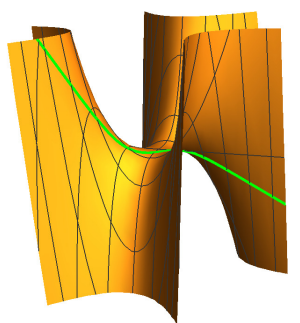

b)
Figure 3. Initial $u$ - and $v$ - parameter curve like a geodesic a) and other geodesic b).

\section{Gaudi's surface}

At Gaudi's surface with parametrization $r(u, v)=\left(u, v, a u \sin \frac{v}{b}\right)$, for parameters $a$ and $b$, initial $v$-parameter curve is a geodesic, while every $u$ - parameter curves are geodesics (fig. 4).

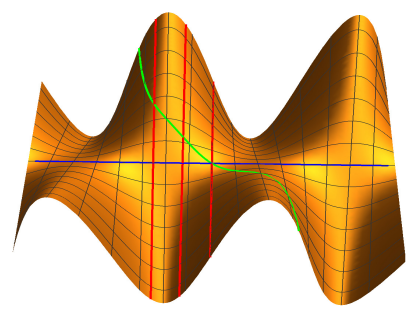

a)

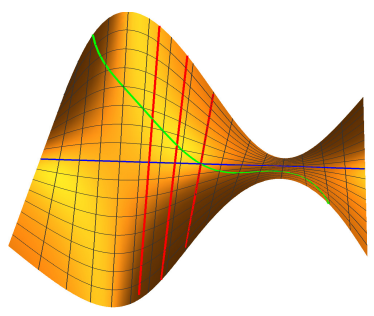

b)
Figure 4. Geodesics on Gaudi's surfaces with parameter $(1,1)$ and $(1,2)$.

\section{Hyperbolic paraboloid}

$u$ - and $v$ - parameter curves on Hyperbolic paraboloid with parametrization $r(u, v)=(u, v, u v)$ are straight lines, and they are also geodesics (fig. 5 a)), while at the surfaces with the parametrization $r(u, v)=\left(u, v, u^{2}-v^{2}\right)$ (fig. $\left.5 \mathrm{~b}\right)$ ) only initial $u$ and $v$-parameter curves are geodesics. 


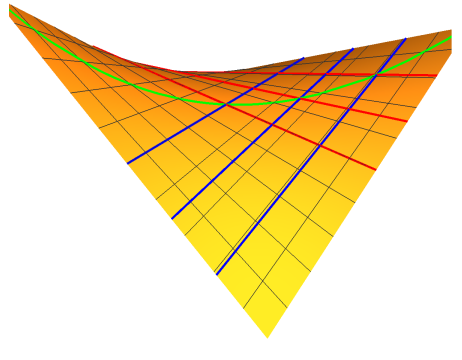

a)

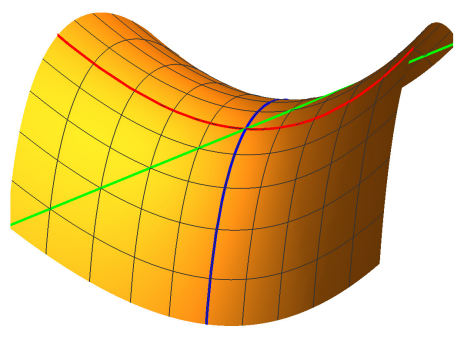

b)
Figure 5. Geodesics on hyperbolic paraboloid.

\section{Developable surfaces}

Firstly we are going to define a regular plane curve

$$
\text { curve }(t)=(x(t), y(t), c), \quad c=\text { const. }
$$

Then, the surface

$$
\operatorname{surface}(u, v)=(x(u), y(u), \operatorname{cg}(u) f(v)), \quad g(u) f(v) \neq 0,
$$

is a surface with $K=0$, i.e. developable surface, that contains the given curve as a geodesic, where $g(u)$ and $f(v)$ are taken arbitrarily. Mean curvature of this surface will be

$$
H=\frac{g^{3}(u) f^{\prime 3}(v)\left(x^{\prime \prime}(u) y^{\prime}(u)-x^{\prime}(u) y^{\prime \prime}(u)\right)}{2\left(g^{2}(u) f^{\prime 2}(v)\left(x^{\prime 2}(u)+y^{\prime 2}(u)\right)\right)^{3 / 2}} .
$$

It is clear that given plane curve represents an initial $u$ - parameter curve, while every $v$-parameter curve is a straight line, and is a geodesic on this surface.

For example, one of surfaces that contains sinusoid as a geodesic can be obtained for $g(u)=\cos u$ and $f(v)=\sin v$ :

$$
\mathcal{S}: \quad r(u, v)=(u, \sin u, \cos u \sin v) .
$$

This surface $\mathcal{S}$ (fig. 6) has curvatures

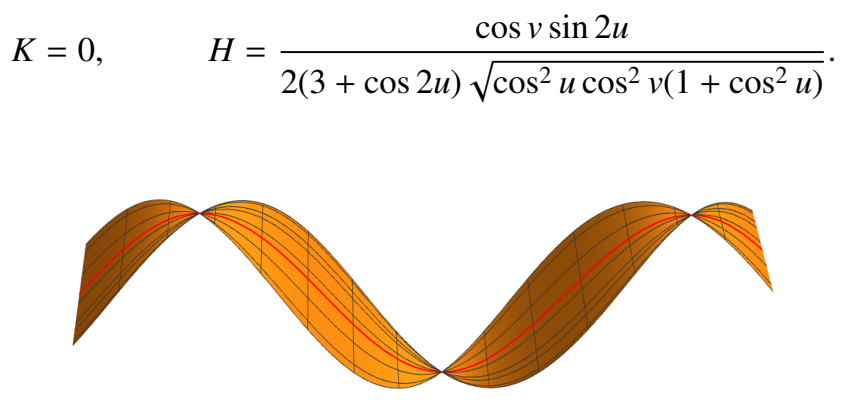

Figure 6. Sinusoid like a geodesic on surface with $K=0$.

\section{Minimal surfaces}

It is well known that some minimal surfaces contain special curves as a geodesics (see Abbena (2006)). For example, the Enneper surface contains the Tschirnhausen cubic as a geodesic and that curve is also initial $u$-parameter curve and $v$-parameter curve of this surface. Also Catalan's surface contains a cycloid as a geodesics and it is its initial $u$ - parameter curve.

The Scherk's surface and the Scherk's fifth surface contains initial $u$ - and $v$-parameter curves as a geodesics (fig. 7).

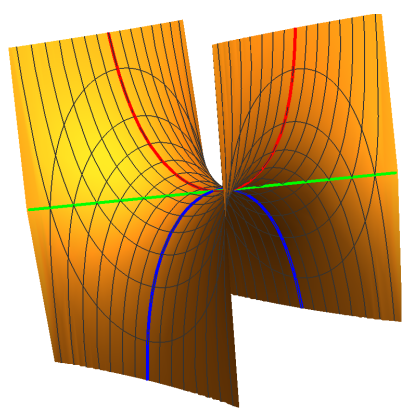

a)

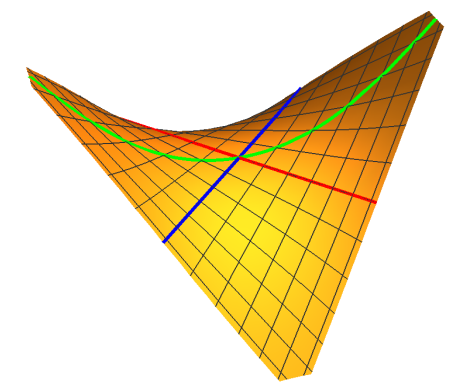

b)
Figure 7. Geodesics on Scherk's surfaces

Considering that the Catenoid is a surface of revolution, its meridians ( $v$ - parameter curves) are geodesics, and only the initial parallel satisfies conditions for geodesics (fig. 8 a)). Since the catenoid is locally isometrical to the helicoid, all its properties are same as those of the helicoid. (fig. 8 b)).

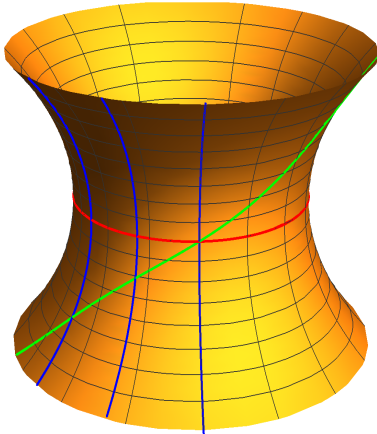

a)

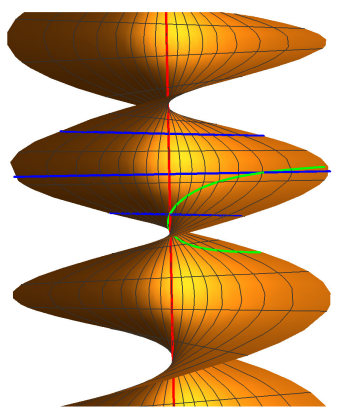

b)
Figure 8. Catenoid and Helicoid

\section{CONSTRUCTION OF THE MINIMAL SURFACES THAT CONTAIN EPICYCLOID AS A GEODESIC}

Construction of the minimal surfaces that contain some curve as a geodesic represents Björling's problem. Construction of these surfaces in Mathematica based on a given curve is represented in Abbena (2006).

One of the simplest Björling's surfaces that contains a known curve as a geodesic is the Catalan's minimal surface (fig. 9). At this surface one of geodesics is cycloid.

Also, at Enneper surface initial $u$ - and $v$-parameter curves are geodesics and represent the Tschirnhausen cubic (fig. 10).

Epicycloid represents a curve that is given as a trail of a determined point of the circle $k_{1}=\left(O_{1}, b\right)$ that spins around the circle $k_{0}=(O, a)$. For different relations of their radii they get 


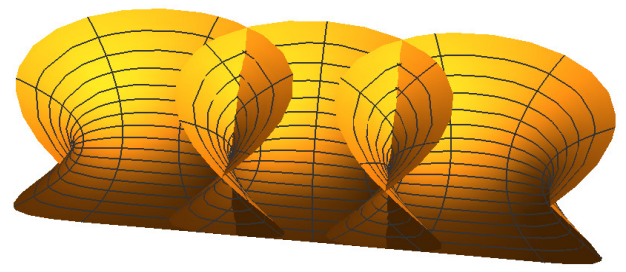

Figure 9. Catalan surface.

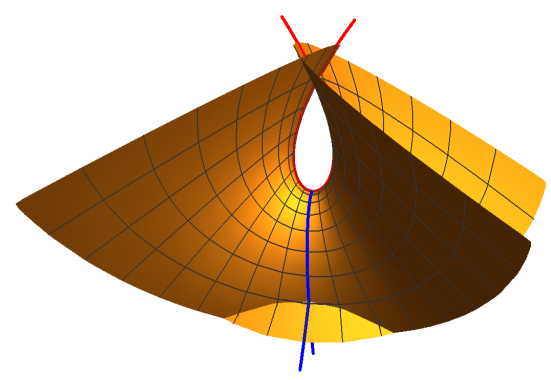

Figure 10. Enneper surface.

different epicycloids. Out of the group of epicycloids, cardioids, nephroids and ranuncloids are best known.

Parametric equations of epicycloid with parameters $a$ and $b$ are:

$$
\left\{\begin{array}{l}
x=(a+b) \cos t-b \cos \left(\frac{a+b}{b} t\right) \\
y=(a+b) \sin t-b \sin \left(\frac{a+b}{b} t\right) .
\end{array}\right.
$$

Parametric equations of the minimal surface that contains arbitrarily epicycloid as a geodesic are:

$$
\left\{\begin{array}{l}
x=(a+b) \cos u \cosh v-b \cos \left(\frac{a+b}{b} u\right) \cosh \left(\frac{a+b}{b} v\right) \\
y=(a+b) \sin u \cosh v-b \sin \left(\frac{a+b}{b} u\right) \cosh \left(\frac{a+b}{b} v\right) \\
z=\frac{4 b(a+b)}{a} \sin \left(\frac{a}{2 b} u\right) \sinh \left(\frac{a}{2 b} v\right) .
\end{array}\right.
$$

We will discuss minimal surfaces obtained on the epicycloid depending on the parameters (values of the parameters are given in the table 1.) Their graphic representation is given on fig. 11, 12, 13,14 and 15.
Table 1. Values of the parameters.

\begin{tabular}{|c|c|cc|}
\hline$[a, b]$ & $t$ & \multicolumn{2}{|c|}{$u, v$} \\
\hline$[1,1]$ & $0 \leq t \leq 2 \pi$ & $0 \leq u \leq 2 \pi$, & $-0.5 \leq v \leq 0.5$ \\
\hline$[2,1]$ & $0 \leq t \leq 2 \pi$ & $0 \leq u \leq 2 \pi$, & $-0.5 \leq v \leq 0.5$ \\
\hline$[5,1]$ & $0 \leq t \leq 2 \pi$ & $0 \leq u \leq 2 \pi$, & $-0.3 \leq v \leq 0.3$ \\
\hline$[7,2]$ & $0 \leq t \leq 4 \pi$ & $0 \leq u \leq 4 \pi$, & $-0.3 \leq v \leq 0.3$ \\
\hline$[3,2]$ & $0 \leq t \leq 4 \pi$ & $0 \leq u \leq 4 \pi$, & $-0.5 \leq v \leq 0.5$ \\
\hline
\end{tabular}
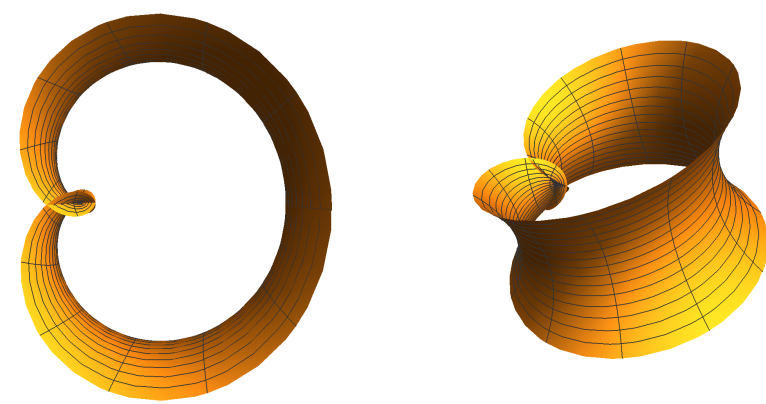

Figure 11. Minimal surface with parameters $[1,1]$.
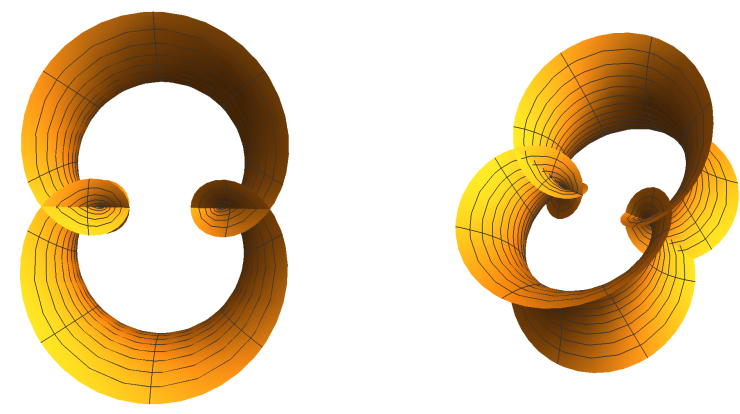

Figure 12. Minimal surface with parameters $[2,1]$.
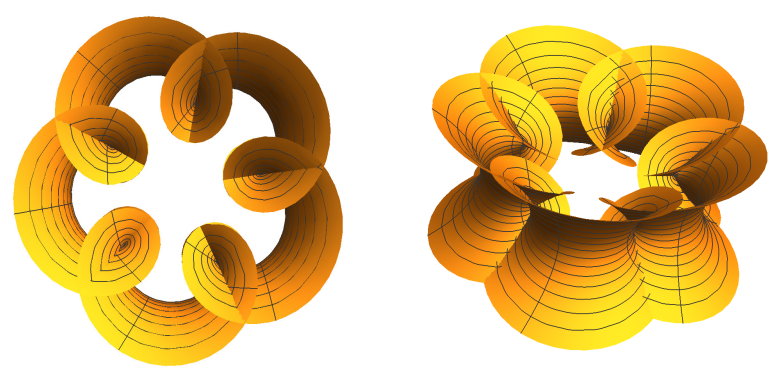

Figure 13. Minimal surface with parameters $[5,1]$.

We see that these surfaces represent "closed Catalan's surfaces". The Gaussian curvature of the minimal surface given for parameters $[2,1]$ is a shown on fig. 16.

For $a<b$ there is a very special group epicycloids that are called pericycloids. Minimal surfaces whose pericycloids are geodesic are shown on fig. 17, 18, 19 and values of the parameters are given in the table 2. 

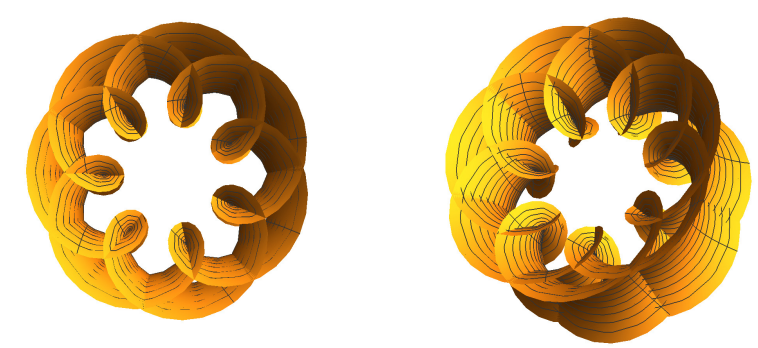

Figure 14. Minimal surface with parameters [7,2].
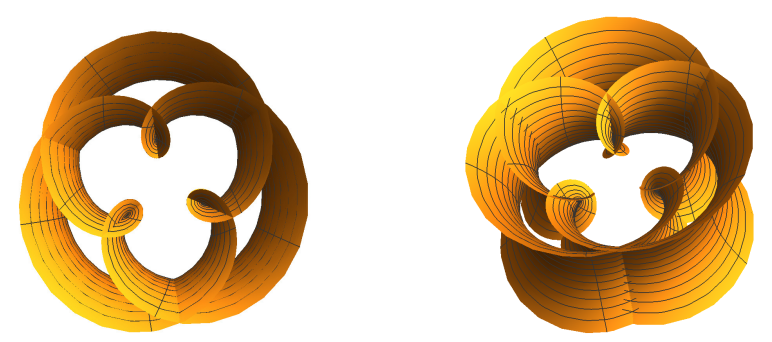

Figure 15. Minimal surface with parameters [3,2].

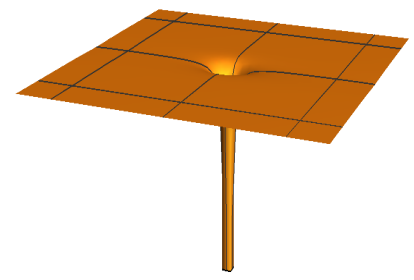

Figure 16. Gaussian curvature of minimal surface with parameters $[2,1]$.

Table 2. Values of the parameters

\begin{tabular}{|c|c|cc|}
\hline$[a, b]$ & $t$ & \multicolumn{2}{|c|}{$u, v$} \\
\hline$[1,2]$ & $0 \leq t \leq 4 \pi$ & $0 \leq u \leq 4 \pi$, & $-0.5 \leq v \leq 0.5$ \\
\hline$[1,3]$ & $0 \leq t \leq 4 \pi$ & $0 \leq u \leq 4 \pi$, & $-0.5 \leq v \leq 0.5$ \\
\hline$[2,3]$ & $0 \leq t \leq 6 \pi$ & $0 \leq u \leq 6 \pi$, & $-0.5 \leq v \leq 0.5$ \\
\hline
\end{tabular}
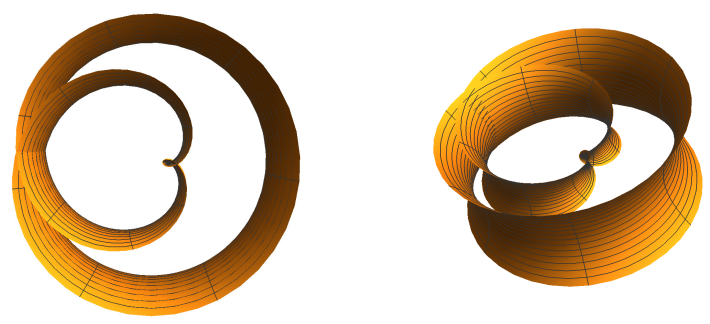

Figure 17. Minimal surface with parameters [1,2].
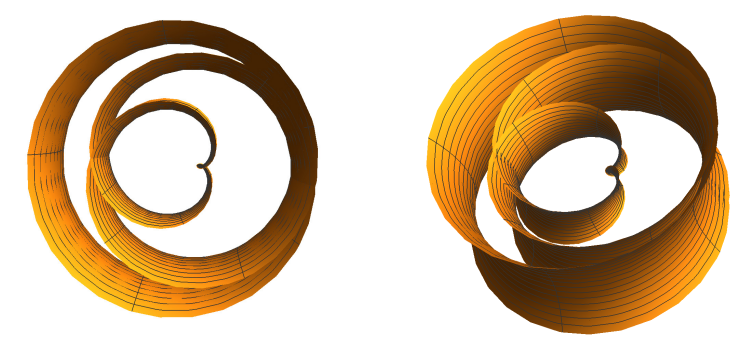

Figure 18. Minimal surface with parameters $[1,3]$.
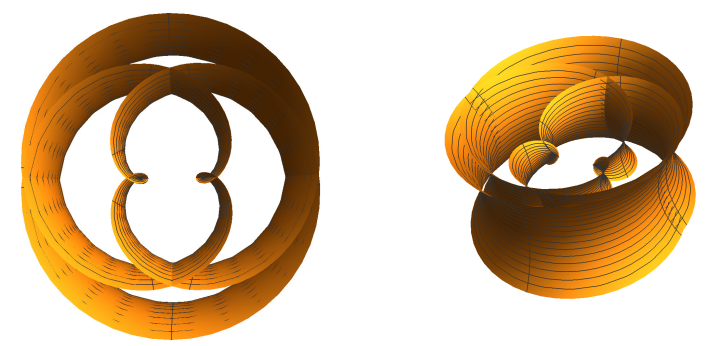

Figure 19. Minimal surface with parameters [2,3].

\section{APPENDIX}

Geodesic vs shortest path

Due to the analogy with the straight lines in plane, geodesics are often connected with the shortest path between two points on the surface. However, its not always possible to identify this terms. As one of the examples, we are going to give the distance between two points $A$ and $B$ on sphere, that belong to a great circle of the sphere and that are not diametrally opposite to each other (fig. 20).

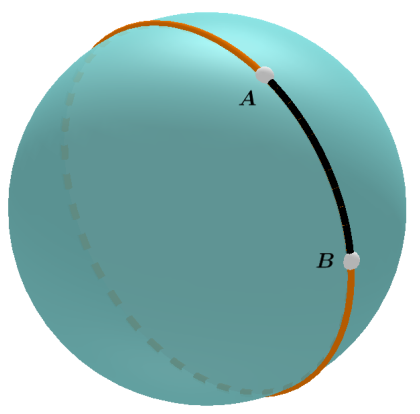

Figure 20. Distance of points on the sphere.

Observer points divide a great circle into two arcs, one of which is shorter than the other. Its clear that both arcs are the parts of the same geodesic, but if points are connected with the longer arc, then it is not the shortest path between them.

\section{ACKNOWLEDGEMENT}

The first author was supported by the research project 174025 of the Serbian Ministry of Science. 


\section{REFERENCES}

Abbena, E., S. S. . G.-A. 2006, Modern differential geometry of curves and surfaces with Mathematica.Boca Raton: Chapman and Hall CRC. 3rd ed.

Abdel-All, N. H., . A.-G. E. I. 2013, Numerical treatment of geodesic differential equations on a surface in $\mathbb{R}^{3}$. International Mathematical Forum, 8, pp. 15-29. doi:10.12988/imf.2013.13003

Do Carmo, M. 1976, Differential Geometry of Curves and Surfaces. Prentice-Hall
Khuangsatung, W. C. P. 2012, Some geodesics in open surfaces classified by Clairauts relation, World Academy of Science. Engineering and Technology, 6(9), pp. 1360-1364.

Lewis, J. 2002, Geodesics Using Mathematica. Rose-Hulman Undergraduate Mathematics Journal, 3(1).

Pressley, A. 2012, Elementary Differential Geometry. London: Springer Science and Business Media LLC. doi:10.1007/9781-84882-891-9

Toponogov, V. 2006, Differential geometry of curves and surfaces. Birkhauser: Boston 